\title{
Mycophenolate Mofetil Improves Exercise Tolerance in Systemic Sclerosis Patients with Interstitial Lung Disease: A Pilot Study
}

\author{
Valentina Vaiarello - Stefano Schiavetto - Federica Foti • \\ Antonietta Gigante - Francesco Iannazzo • Gregorino Paone • \\ Paolo Palange $\cdot$ Edoardo Rosato (D)
}

Received: July 28, 2020 / Published online: August 29, 2020

(C) The Author(s) 2020

\begin{abstract}
Introduction: Systemic sclerosis (SSc) is an autoimmune disease characterized by the overproduction of collagen leading to fibrosis of the skin and internal organs. Interstitial lung disease (ILD) is one of the major causes of death in patients with SSc. Exercise tolerance can be investigated by cardio-pulmonary exercise testing (CPET). First-line therapies in patients with SSc associated with ILD (SSc-ILD) include cyclophosphamide and mycophenolate mofetil (MMF). The aim of this study was to evaluate the response of patients with SSc-ILD to MMF by means of CPET.
\end{abstract}

Digital Features To view digital features for this article go to https://doi.org/10.6084/m9.figshare.12821708.

V. Vaiarello · F. Foti · A. Gigante - F. Iannazzo ·

E. Rosato $(\square)$

Department of Translational and Precision

Medicine, Sapienza University, Rome, Italy

e-mail: edoardo.rosato@uniroma1.it

S. Schiavetto - G. Paone - P. Palange

Department of Public Health and Infectious

Diseases, Lung Function and Exercise Section,

Sapienza University, Rome, Italy
Methods: Ten consecutive SSc patients were enrolled in this study. All SSc patients underwent clinical evaluation, echocardiography, pulmonary function tests, high-resolution computed tomography (HRCT) and CPET at baseline and after 2 years of therapy with MMF. Results: After 24 months of treatment with MMF (target dose $1500 \mathrm{mg}$ twice daily), forced vitality capacity, diffusing capacity of the lungs for carbon monoxide and systolic pulmonary arterial pressure had not improved significantly and there were no significant differences in HRCT findigns. In addition, peak oxygen uptake $\left(V^{\prime} \mathrm{O}_{2}\right.$ peak) and ventilatory equivalents for carbon dioxide production $\left(V^{\prime} E / V^{\prime} \mathrm{CO}_{2}\right.$ slope) had not improved significantly. In contrast, there was a significant improvement from baseline to 24 months of treatment in the respiratory exchange ratio [median (interquartile range): $1.07(0.92-1.22)$ vs. $1.26(1.22-1.28)$, respectively; $p<0.01]$ and in the Borg scale for leg discomfort [median (interquartile range): 5 (5-7) vs. 4 (3-4), respectively; $p<0.01$ ] .

Conclusion: These data from our pilot study on a small cohort of SSc patients are the first to demonstrate that treatment with MMF can improves exercise tolerance and leg discomfort in patients with SSc-ILD. These preliminary results need to be confirmed in large randomized studies. 
Keywords: Cardio-pulmonary exercise testing; Interstitial lung disease; Mycophenolate mofetil; Systemic sclerosis

\section{Key Summary Points}

Interstitial lung disease (ILD) is the major complication of systemic sclerosis (SSc).

Response of patients with SSc associated with ILD (SSc-ILD) to immunosuppressive therapy was assessed by pulmonary function tests.

Data are currently lacking on the exercise tolerance of SSc patients with ILD who have undergone standard immunosuppressive therapy.

In this small pilot study, the exercise tolerance of SSc patients with ILD was assessed by cardio-pulmonary exercise testing after 24 months of standard immunosuppressive therapy.

Mycophenolate mofetil improved exercise tolerance in this small cohort of patients with SSc-ILD.

\section{DIGITAL FEATURES}

This article is published with digital features to facilitate understanding of the article. You can access the digital features on the article's associated Figshare page. To view digital features for this article go to https://doi.org/10.6084/m9. figshare.12821708.

\section{INTRODUCTION}

Systemic sclerosis (SSc) is an autoimmune disease characterized by immune dysregulation, vasculopathy and the overproduction of collagen leading to skin and internal organ fibrosis [1]. Pulmonary involvement is frequent among patients with SSc, and it most usually manifests as interstitial lung disease (ILD) and/or pulmonary vasculopathy, leading to pulmonary arterial hypertension (PAH) [2]. Systemic sclerosis associated interstitial lung disease (SScILD) is a complex process involving inflammation, alveolar epithelial damage and the activation of resident fibroblasts, resulting in thickening of the pulmonary interstitium [3]. Both pulmonary fibrosis and PAH are major causes of death in SSc patients [4].

Therapeutic strategies for the treatment of SSc-ILD include the immunosuppressants cyclophosphamide and mycophenolate mofetil (MMF) as first-line therapies, with MMF the most commonly used of the two medications because it seems to be better tolerated. The standard MMF therapeutic regimen consists of an initial dose of $500 \mathrm{mg}$ twice daily, increased monthly, until a maximum dose of $1.5 \mathrm{~g}$ twice daily is reached, administered for 24 months [5-8]. Due to the potential progressive fibrosing phenotype of SSc-ILD and the risk of rapid deterioration in the patient's health, particularly evident in the first 3 years of disease onset, effective and early disease monitoring is extremely important [9].

Disease progression and response to treatment are usually assessed based on the evolution of respiratory symptoms, pulmonary function tests (PFTs), diffusing capacity of the lungs for carbon monoxide (DLco), high-resolution computed tomography (HRCT) findings, echocardiography with systolic pulmonary arterial pressure (sPAP) and exercise tolerance [10]. In particular, exercise tolerance may be investigated with cardio-pulmonary exercise testing (CPET). To date, there are no clinical data in the literature on the use of CPET to evaluate the response of patients with SSc-ILD to standard therapy. In patients with ILD, CPET may be particularly useful for detecting exercise-related ventilatory and gas-exchange abnormalities early in the course of the disease at a time when resting lung-function measurements appear to be normal. Perhaps more importantly, arterial desaturation and other CPET indices have proven useful in predicting the prognosis of patients with ILD, and this has also been shown in patients with SSc-ILD [11-13]. 
Recently, nintedanib has shown to reduce the rate of decline of forced vital capacity (FVC) in patients with SSc-ILD over 1-year period [14].

CPET has been used to assess patients undergoing right heart catheterization (RHC) and is included among the parameters to estimate risk in the European Society of Cardiology (ESC) and European Respiratory Society (ERS) guidelines $[15,16]$.

The aim of this study was to determine if treatment with MMF improves exercise tolerance in patients with SSc-ILD.

\section{METHODS}

\section{Participants}

Ten consecutive patients meeting the American College of Rheumatology/European League Against Rheumatism criteria for the classification and diagnosis of SSc criteria were enrolled in this prospective study [17].

In accordance with Scleroderma Lung Study II (SLS II) [7], participants were all adults (age range 18-75 years) with SSc-ILD, either as limited SSc (lcSSc) or diffuse cutaneous SSc (dcSSc) with active ILD, defined as the presence of both a restrictive to borderline restrictive ventilatory impairment [FVC (predicted forced vital capacity) of $<80-85 \%$ but $\geq 45 \%$ ] and the presence of any ground glass opacity on HRCT. Participants also had to have exertional dyspnea and a disease duration of $\leq 7$ years from the onset of the first non-Raynaud's symptom of SSc [7].

Patients with pulmonary disease not related to SSc, evidence of significant airflow obstruction, PAH, chronic heart failure, cardiac arrhythmias and conduction disorders, history of uncontrolled systemic hypertension, valvular heart diseases, anemia, myositis and neuromuscular diseases were excluded from the study, as were smokers and pregnant or breastfeeding women.

\section{Clinical Assessments}

Data on age, disease duration and modified Rodnan Skin Score (mRSS) were systematically collected. Nailfold videocapillaroscopy was performed using a videocapillaroscope (Pinnacle Studio Version 8; Pinnacle Systems, Inc., Mountain View, CA, USA) equipped with a $500 \times$ optical probe. In accordance with Cutolo et al., the patterns identified within the "SSc pattern" were early, active and late [18].

\section{Pulmonary Function Tests}

Spirometric parameters of flows and volumes [FEV1 (forced expiratory volume in the first second), FVC, FEV1/FVC)] and DLco, corrected for hemoglobin concentration, were recorded with a Quark PFT 2 spirometer (Cosmed, Rome, Italy) and expressed according to the standards recommended by the American/European Respiratory Society $[19,20]$. All spirometric parameters are expressed as the percentage of predicted value.

\section{High-Resolution Computed Tomography}

High-resolution computed tomography was performed according to standard protocol using a CT 64GE light Speed VCT power scanner (model Somatom Definition; Siemens AG, Forcheim, Germany) at a voltage of $120 \mathrm{kV}$ and modulating the tube current levels to the scanning volume to reduce the dose. A quantitative score of HRCT was obtained according to the SLS II study [17]. According to the HRCT results, all patients had a quantitative score of ILD > $25 \%$ with the presence of ground-glass areas [7].

\section{Cardiopulmonary Exercise Test}

An incremental symptom-limited CPET was performed on an electronically braked cycloergometer (Ergoline-800; Mortara, Bologna, Italy), according to a standardized protocol and following international guidelines. The individual was attached to the breath-by-breath lung gas exchange system using a mask and breathing through a bidirectional turbine mass flow sensor (Quark PFT; Cosmed, Rome, Italy). The exercise protocol consisted of $3 \mathrm{~min}$ of rest and $3 \mathrm{~min}$ of unloaded cycling, followed by an 
incremental work rate to induce voluntary exhaustion in about $10 \mathrm{~min}$, followed by $3 \mathrm{~min}$ of recovery. Electrocardiography (ECG) and pulse oximetry were continuously monitored, and blood pressure was measured every $2 \mathrm{~min}$. The use of calcium channel blockers, endotelin1 receptor antagonist and phosphodiesterase type 5 inhibitors was discontinued $72 \mathrm{~h}$ before the CPET, and the CPET were performed $24 \mathrm{~h}$ prior the next infusion of Iloprost. Tidal volume, respiratory rate, heart rate (HR) and lung gases exchange $\left(\mathrm{O}_{2}, \mathrm{CO}_{2}\right)$ were measured breath-by-breath continuously during the entire test test. The consumption rate of oxygen $\left(V^{\prime} \mathrm{O}_{2}\right)$ and carbon dioxide $\left(V^{\prime} \mathrm{CO}_{2}\right)$, the $V^{\prime} \mathrm{O}_{2} / \mathrm{HR}$ ratio, peak oxygen uptake $\left(V^{\prime} \mathrm{O}_{2}\right.$ peak $)$ and the respiratory exchange ratio $\left[V^{\prime} \mathrm{CO}_{2} / V^{\prime} \mathrm{O}_{2}\right.$ (RER)] were computed and averaged every $10 \mathrm{~s}$. The lactic threshold was determined by the V-slope method. The ventilatory equivalents for carbon dioxide production $\left(V^{\prime} E / V^{\prime} \mathrm{CO}_{2}\right.$ slope) was calculated as the slope of the linear relationship between pulmonary ventilation (VE) and $V^{\prime} \mathrm{CO}_{2}$ from 1 min after the beginning of loaded exercise to the end of the isocapnic buffering period. A submaximal test is defined by RER $\leq 1.05$ [11].

Clinical evaluation results and PFTs were assessed at baseline and every 6 months during the study, whereas ECG was assessed at baseline and every 12 months during the study. HRCT and CPET were performed and the results assessed at baseline and at 24 months.

\section{Statistical Analysis}

The coefficient of skewness and kurtosis with the Shapiro-Wilk test were used to evaluate the normal distribution of data. All results were expressed as the median and interquartile range (IQR). SPSS version 25.0 software (IBM Corp., Armonk, NY, USA) was used for the statistical analysis. Group comparisons were made using the Mann-Whitney test. Spearman's rank correlation coefficient was used to test for an association between numerical variables. The Fisher's exact test was used to compare categorical variables. $p$ values $<0.05$ were considered to be significant.

\section{Compliance with Ethics Guidelines}

The ethics committee of Sapienza University approved the study (No. 5435). The study was performed in accordance with the Helsinki Declaration of 1964, and its later amendments. All subjects provided informed consent to participate in the study.

\section{RESULTS}

\section{Baseline Findings}

Ten SSc patients [9 females; median age 53 (IQR 43-58) years] were enrolled in the study. All patients had dcSSc. The epidemiological and

Table 1 Epidemiological and clinical features of the patients with systemic sclerosis enrolled in the study

\begin{tabular}{ll}
\hline Epidemiological and clinical features & Values \\
\hline Female, $n$ (\%) & $9(90 \%)$ \\
Age, years & $53(43-58)$ \\
Disease duration, years & $5(4-6)$ \\
mRSS & $17(16-22)$ \\
BMI, kg/m ${ }^{2}$ & $22.4(21.3-23.2)$ \\
dcSSc, $n$ (\%) & $10(100 \%)$ \\
Digital ulcers history, $n(\%)$ & $6(60 \%)$ \\
SSc-specific autoantibodies, $n(\%)$ & \\
Scl70 & $9(90 \%)$ \\
Anticentromere & 0 \\
None & $1(10 \%)$ \\
Capillaroscopic pattern, $n(\%)$ & \\
Early & 0 \\
Active & $1(10 \%)$ \\
Late & $9(90 \%)$ \\
\hline
\end{tabular}

Unless indicated otherwise, values are presented as the median with the interquartile range (IQR) in parenthesis $A C A$ Anticentromere antibodies, $B M I$ body mass index, $d c S S c$ diffuse cutaneous systemic sclerosis, $m R S S$ modified Rodnan skin score, $S c l 70$ antitopoisomerase I antibodies, $S S c$ systemic sclerosis 
clinical features of all ten patients are shown in Table 1. At baseline, the median values (IQR) of FVC, DLco and sPAP were 73\% (71-76\%) of the predicted value, $68 \%(65-69 \%)$ of the predicted value and 28 (27-29) $\mathrm{mmHg}$, respectively. Also at baseline, the median values (IQR) of the CPET parameters were: RER, $1.07(0.92-1.22) ; V^{\prime} \mathrm{O}_{2}$ peak, $80 \%$ (76-87\%) of the predicted value; $V^{\prime} E /$ $V^{\prime} \mathrm{CO}_{2}$ slope, 29.8 (25.2-35.4); and leg discomfort Borg scale, 5 (5-7). Five (50\%) patients performed a submaximal test, as demonstrated by RER $<1.05$. HRCT showed a median score of $30 \%(25-38 \%)$ of all parenchymal lung with ground glass areas. Results from the PFTs, sPAP and CPET are shown in Table 2.

\section{After Treatment with MMF}

Mycophenolate mofetil (target dose $1500 \mathrm{mg}$ twice daily) was administered for 24 months. The post-therapy assessment showed that FVC, DLco (both presented as percentage of predicted value) and SPAP, all presented as the median (IQR) of all measurements during the follow-up, did not improve significantly following therapy

Table 2 Results of pulmonary function tests, echocardiography and cardiopulmonary exercise testing pre- and post-therapy with mycophenolate mofetil

\begin{tabular}{|c|c|c|}
\hline PFTs, echocardiograpy and CPET & Pre-therapy with MMF & Post-therapy witl \\
\hline \multicolumn{3}{|l|}{ Variables of PFTs and ecocardiography } \\
\hline FVC, $\%$ of predicted value & $73(71-76)$ & $76.5(70-79)$ \\
\hline DLco, $\%$ of predicted value & $68(65-69)$ & $66(65-68)$ \\
\hline sPAP, mmHg & $28(27-29)$ & $28(27-29)$ \\
\hline \multicolumn{3}{|l|}{ Variables of CPET } \\
\hline RR peak & $35.3(33-42)$ & $37.6(33.9-39.1)$ \\
\hline RER & $1.07(0.92-1.22)$ & $1.26(1.22-1.28)$ \\
\hline Watt peak, W & $65(60-70)$ & $65(60-78)$ \\
\hline$V^{\prime} \mathrm{O}_{2}$ peak, $\mathrm{ml} / \mathrm{min}$ & $987(933-1381)$ & $1195(987-1230)$ \\
\hline $\mathrm{VO}_{2}, \mathrm{ml} / \mathrm{min} / \mathrm{kg}$ & $16.6(16.3-19)$ & $17.6(14.5-19.8)$ \\
\hline$V^{\prime} \mathrm{O}_{2}$ peak, $\%$ & $80(76-87)$ & $88.5(83-94)$ \\
\hline$V^{\prime} E / V^{\prime} \mathrm{CO}_{2}$ slope & $29.8(25.2-35.4)$ & $26(25-30.3)$ \\
\hline HR peak, bpm & $138.5(128-168)$ & $133.5(119-168)$ \\
\hline Basal SBP, mmHg & $115(70-130)$ & $110(70-125)$ \\
\hline Peak SBP, mmHg & $170(155-175)$ & $165(150-170)$ \\
\hline Dyspnea Borg scale & $5(3-7)$ & $5(3-5)$ \\
\hline Leg discomfort, Borg scale & $5(5-7)$ & $4(3-4)$ \\
\hline
\end{tabular}

All values in table are presented as the median with the IQR in parenthesis. Post-therapy FVC, DLco and sPAP values are median values of measurements taken during the 2-year follow-up

CPET Cardiopulmonary exercise testing, DLco single-breath carbon monoxide diffusing capacity, $F V C$ forced vital capacity, $H R$ heart rate, $M M F$ mycophenolate mofetil, PFTs pulmonary function tests, $s P A P$ systolic pulmonary artery pressure, $R E R$ respiratory exchange ratio, $R R$ respiratory rate, $S B P$ systolic blood pressure, $V^{+} E / V^{\top} C O_{2}$ ventilatory equivalents for carbon dioxide production, $V^{\prime} O_{2 p e a k}$ peak oxygen uptake 
[FVC: $73(71-76)$ vs. 76.5 (70-79), $p>0.05$; DLco: 68 (65-69) vs. 66 (65-68), $p>0.05$; sPAP: 28 (27-29) vs. 28 (27-29) $\mathrm{mmHg}, p>0.05]$ (Table 2). In addition, no significant differences in the HRCT score were reported after 2 years of therapy with MMF [31\% (24-39\%)], and there were no significant improvements in the $V^{\prime} \mathrm{O}_{2}$ peak $\left[\begin{array}{lllll}80 \% & (76-87 \%) & \text { vs. } 88.5 \% & (83-94 \%) \text {; }\end{array}\right.$ $p>0.05]$ and $V^{\prime} E / V^{\prime} \mathrm{CO}_{2}$ slope [29.8 (25.2-35.4) vs. 26 (25-30.3), $p>0.05]$. In contrast, a significant improvement of RER [1.07 (0.92-1.22) vs $1.26(1.22-1.28) ; p<0.01]$ and the leg discomfort Borg scale [5 (5-7) vs. $4(3-4), p<0.01]$ was observed (Table 2; Fig. 1). All patients performed a maximal test as demonstrated by RER $>1.05$.

\section{DISCUSSION}

Our results demonstrate that MMF therapy improves FVC and DLco in patients with SSc-
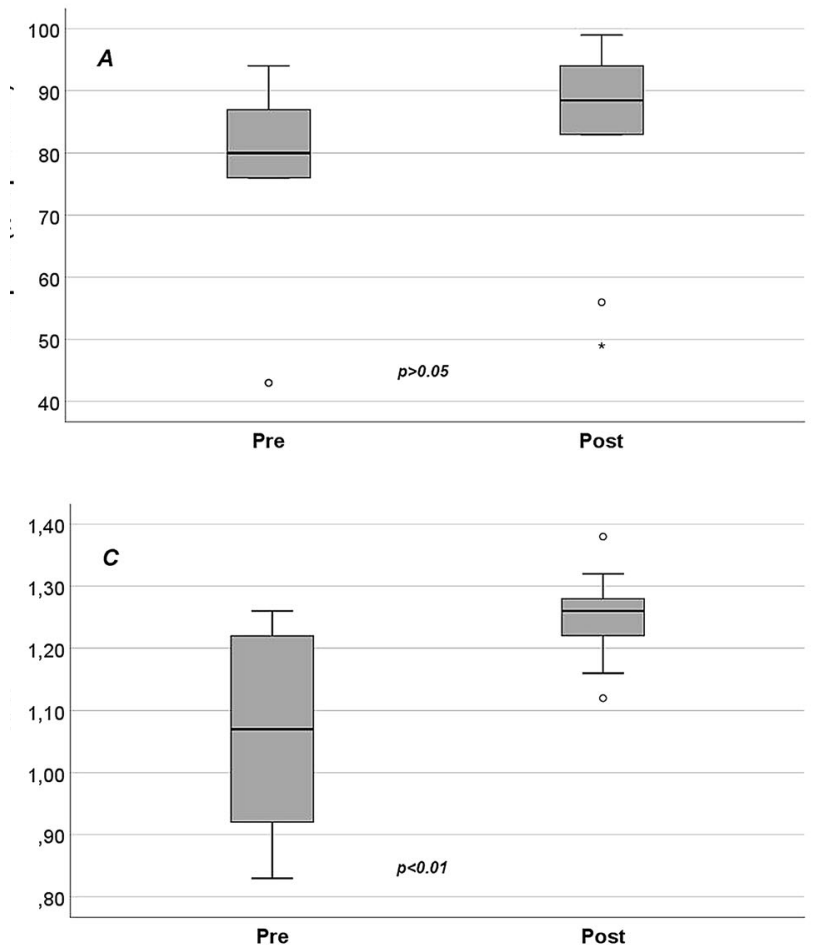

Fig. 1 Box plot of cardiopulmonary exercise test parameters pre- and post-therapy with mycophenolate mofetil. a Peak oxygen uptake $\left(V^{\prime} \mathrm{O}_{2}\right.$ peak, b ventilatory equivalents for
ILD. This study shows, for the first time, that MMF therapy can improve exercise tolerance, as assessed by CPET, in this patient population.

In the SLS I and SLS II studies, both cyclophosphamide and MMF were found to significantly improve FVC and DLco in patients with scleroderma lung disease. The authors of these trials concluded that MMF significantly reduced the progression of ILD for up to 1 year after cessation of immunosuppressive therapy; at $>1$ year, the ILD-SSc worsened with concomitant worsening of PFTs. The results of our study in a small sample of SSc patients confirm those of these large randomized trials $[5,6]$.

Our study shows for the first time that therapy improves exercise tolerance by demonstrating the MMF therapy improves both oxygen consumption $\left(V^{\prime} \mathrm{O}_{2}\right.$ peak) and also $V^{\prime} E /$ $V^{\prime} \mathrm{CO}_{2}$ (mismatch). One possibility is that immunosuppressive therapy reduces inflammation (ground glass) and allows both better oxygenation and a redistribution of perfusion by
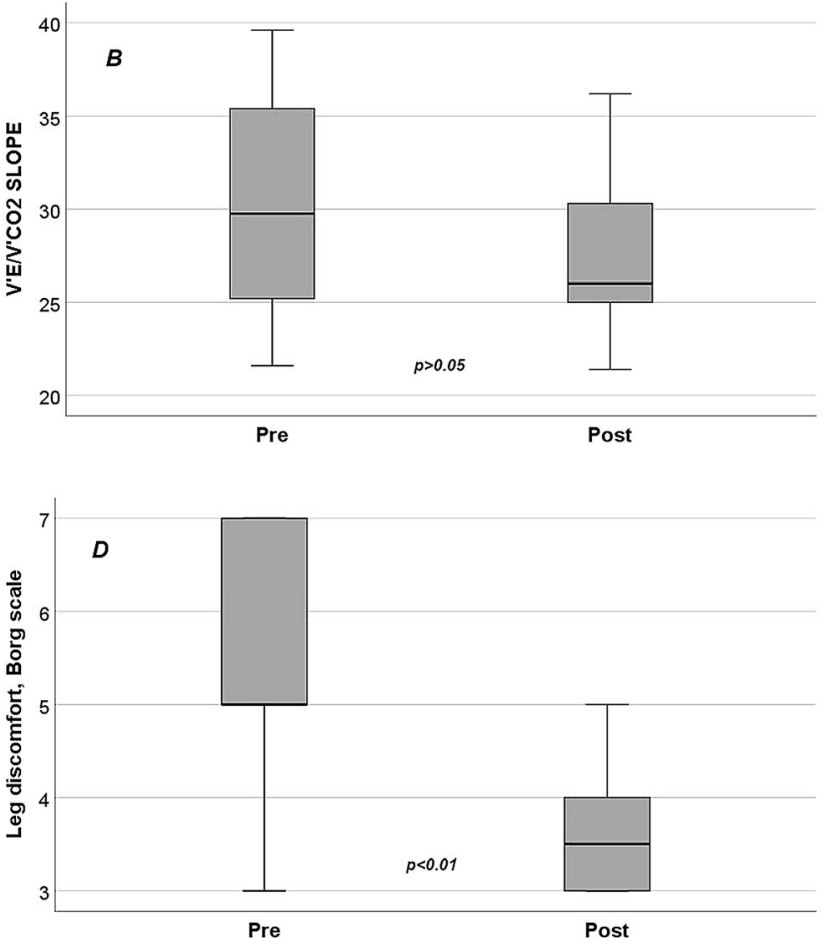

carbon dioxide production $\left(V^{\prime \prime} E / V^{\prime} \mathrm{CO}_{2}\right.$ slope), c respiratory exchange ratio $(R E R)$, d leg discomfort Borg scale 
reducing respiratory mismatch. A reduction of inflammation would allow oxygenation of previously perfused but unventilated areas. Rosato et al. showed that pulmonary vasculopathy occurs independently of fibrosis [12]. We hypothesize that the improvement in ventilation perfusion mismatch is due to a reduction of inflammation since the sPAP values remained constant.

Our data also demonstrate that standard immunosuppressive therapy improves the ability of the patient to perform maximal CPET. Before therapy five patients performed a submaximal test; after therapy no patient performed a submaximal test, as demonstrated by RER $>1.05$. The lower test results prior to testing were caused by both respiratory and muscular discomfort. After immunosuppressive therapy the respiratory dyspnea scale remained constant, with a slight but not significant improvement. Conversely, we observed a significant improvement in the leg discomfort Borg scale. These results suggest that, in the absence of any muscle causes both at baseline and after therapy (e.g. myositis), better muscle oxygenation with a reduction of lactic acidosis is responsible for the reduction in the leg discomfort Borg scale. In addition, improved exercise tolerance, assessed by CPET, corresponds to real-life exercise tolerance with stabilization of the dyspnea Borg scale.

The main limitations of our study are the small sample size and the lack of CPET data at 12 months of follow-up. Despite the limitations of the study, the data are in agreement with those reported in the literature, which demonstrate a functional improvement in patients with ILD-SSc after treatment with MMF.

\section{CONCLUSIONS}

For the first time, we have demonstrated in a small cohort of SSc patients that treatment with MMF improves exercise tolerance and leg discomfort in patients with SSc-ILD. The preliminary data of this small pilot study needs to be confirmed in large randomized studies.

\section{Acknowledgements}

Funding. No funding or sponsorship was received for this study or publication of this article.

Authorship. All named authors meet the International Committee of Medical Journal Editors (ICMJE) criteria for authorship for this article, take responsibility for the integrity of the work as a whole, and have given their approval for this version to be published.

Disclosures. Valentina Vaiarello, Stefano Schiavetto, Federica Foti, Antonietta Gigante, Francesco Iannazzo, Gregorino Paone, Paolo Palange and Edoardo Rosato have nothing to disclose.

Compliance with Ethics Guidelines. The ethics committee of Sapienza University approved the study (No. 5435). The study was performed in accordance with the Helsinki Declaration of 1964, and its later amendments. All subjects provided informed consent to participate in the study.

Data Availability. All data generated or analyzed during this study are included in this published article.

Open Access. This article is licensed under a Creative Commons Attribution-NonCommercial 4.0 International License, which permits any non-commercial use, sharing, adaptation, distribution and reproduction in any medium or format, as long as you give appropriate credit to the original author(s) and the source, provide a link to the Creative Commons licence, and indicate if changes were made. The images or other third party material in this article are included in the article's Creative Commons licence, unless indicated otherwise in a credit line to the material. If material is not included in the article's Creative Commons licence and your intended use is not permitted by statutory regulation or exceeds the permitted use, you will need to obtain permission directly from the copyright holder. To view a copy of this licence, visit http://creativecommons.org/licenses/bync/4.0/. 


\section{REFERENCES}

1. Roofeh D, Jaafar S, Vummidi D, Khanna D. Management of systemic sclerosis-associated interstitial lung disease. Curr Opin Rheumatol. 2019;31:241-9.

2. Wells AU, Steen V, Valentini G. Pulmonary complications: one of the most challenging complications of systemic sclerosis. Rheumatology (Oxford). 2009;48(Suppl 3):40-4.

3. Wells AU. Interstitial lung disease in systemic sclerosis. Press Med. 2014;43:329-43.

4. Tyndall AJ, Bannert B, Vonk M, et al. Causes and risk factors for death in systemic sclerosis: a study from the EULAR Scleroderma Trials and Research (EUSTAR) database. Ann Rheum Dis. 2010;69: 1809-15.

5. Tashkin DP, Elashoff R, Clements PJ, et al. Cyclophosphamide versus placebo in scleroderma lung disease. N Engl J Med. 2006;354:2655-66.

6. Tashkin DP, Roth MD, Clements PJ, et al. Mycophenolate mofetil versus oral cyclophosphamide in scleroderma-related interstitial lung disease (SLS II): a randomised controlled, doubleblind, parallel group trial. Lancet Respir Med. 2016;4:708-19.

7. Volkmann ER, Tashkin DP, LeClair H, et al. Treatment with mycophenolate and cyclophosphamide leads to clinically meaningful improvements in patient-reported outcomes in scleroderma lung disease: results of Scleroderma Lung Study II. ACR Open Rheumatol. 2020;2:362-70.

8. Fernández-Codina A, Walker KM, Pope JE, Scleroderma Algorithm Group. Treatment algorithms for systemic sclerosis according to experts. Arthritis Rheumatol. 2018;70:1820-8.

9. Suliman S, Al Harash A, Roberts WN, Perez RL, Roman J. Scleroderma-related interstitial lung disease. Respir Med Case Rep. 2017;22:109-12.

10. Fischer A, Patel NM, Volkmann ER. Interstitial lung disease in systemic sclerosis: focus on early detection and intervention. Open Access Rheumatol. 2019;11:283-307.

11. ERS Task Force, Palange P, Ward SA, et al. Recommendations on the use of exercise testing in clinical practice. Eur Respir J. 2007;29:185-209.
12. Rosato E, Romaniello A, Magrì D, et al. Exercise tolerance in systemic sclerosis patients without pulmonary impairment: correlation with clinical variables. Clin Exp Rheumatol. 2014;32(6 Suppl 86):S-103-8.

13. Ewert R, Ittermann T, Habedank D, et al. Prognostic value of cardiopulmonary exercise testing in patients with systemic sclerosis. BMC Pulm Med. 2019;19:230.

14. Distler O, Highland KB, Gahlemann M, et al. Nintedanib for systemic sclerosis-associated interstitial lung disease. N Engl J Med. 2019;380:2518-28.

15. Santaniello A, Casella R, Vicenzi M, et al. Cardiopulmonary exercise testing in a combined screening approach to individuate pulmonary arterial hypertension in systemic sclerosis. Rheumatology (Oxford). 2020;59:1581-6.

16. Galiè N, Humbert M, Vachiery JL, et al. 2015 ESC/ ERS Guidelines for the diagnosis and treatment of pulmonary hypertension: the Joint Task Force for the Diagnosis and Treatment of Pulmonary Hypertension of the European Society of Cardiology (ESC) and the European Respiratory Society (ERS): endorsed by: Association for European Paediatric and Congenital Cardiology (AEPC), International Society for Heart and Lung Transplantation (ISHLT). Eur Heart J. 2016;37:67-119.

17. van den Hoogen F, Khanna D, Fransen J, et al. Classification criteria for systemic sclerosis: an American College of Rheumatology/European League Against Rheumatism collaborative initiative. Arthritis Rheum. 2013;65:2737-47.

18. Cutolo M, Sulli A, Secchi ME, Paolino S, Pizzorni C. Nailfold capillaroscopy is useful for the diagnosis and follow-up of autoimmune rheumatic diseases. A future tool for the analysis of microvascular heart involvement? Rheumatology (Oxford). 2006;45(Suppl 4):43-6.

19. Schwaiblmair M, Behr J, Fruhmann G. Cardiorespiratory responses to incremental exercise in patients with systemic sclerosis. Chest. 1996;110: 1520-5.

20. Miller MR, Crapo R, Hankinson J, et al. ATS/ERS Task Force. General considerations for lung function testing. Eur Respir J. 2005;26:153-61. 